\title{
Facial Animation System Design based on Image Processing DU Xueyan ${ }^{1, a}$ \\ ${ }^{1}$ Foreign Language School, Wuhan Polytechnic, Wuhan 430074, P.R China \\ aduxueyanwh@126.com
}

Keywords: Facial Animation, Cartoon Portrait, AAM, Image Processing

\begin{abstract}
Using computer to make face model, establish facial expression animation and exaggerate facial image processing is a hot research topic in the current, which technology has a broad application prospect and huge economic benefits. For example, remote teaching, film and television entertainment, video games and videophone could use the technology. From the current domestic and foreign research related to the subject, there still exists many problems to study because of the complexity of the facial expression.
\end{abstract}

\section{Facial feature recognition and animation generation}

\section{Facial recognition}

Face recognition is one of the basic problems of computer vision, which also belongs to a branch of bio-metrics. It has been widely used in the security and identity recognition, video conference, image and video retrieval based on object, entertainment and other fields. Special position is capable of detecting human face, extract face feature to save source data for facial animation. therefore, the research of facial recognition technology before the facial animation generation algorithm is very necessary. [1]Almost all of the pattern recognition theory and technology has been applied in the field of face recognition, but some special algorithm of face recognition is also developed.

\section{Facial animation generation}

Facial animation generation has a variety of methods, can be roughly divided into facial animation based on 2D model generation and model based on 3D facial animation. In various methods of the model, in addition to the methods such as muscle model is unique to the 3D. Most of the methods have corresponding model of 3D and 2D version, such as morphing algorithm, grid algorithm, the radial basis function (RBF) algorithm.

\section{Texture synthesis and image fusion}

Texture synthesis technology has natural contact face with facial animation generation. Many of the elements can be regarded as a texture, such as facial expressions, wrinkles, skin color changes, and so on. In face recognition based on image, AAM model need to do face global texture statistics and analysis. Condensation algorithm also can build model on the current image texture sampling observation. In facial animation generation of relevant technical, texture synthesis application are more wide. It is visible that the texture synthesis is of positive significance for facial animation generation technology improvement.

\section{Facial feature points localization algorithm}

The extracting and positioning of facial feature points have important applications in the man-machine interface and artificial intelligence. [2]Computer is expected to reach "human nature", change from passive interaction "machine first, people obey machine" to become a machine that can take the initiative to recognize faces, learn by watching, listening, learning the computer, can use natural language to communicate with humans. In this respect, facial features is as the main mode of video input. "Look" of the robot require computer able to identify and distinguish the characteristics, this chapter focuses on several kinds of algorithms of facial feature points localization based on statistics. 


\section{Definition of facial feature points by MPEG • 4}

For the front of the face image, there is a general sense of the "three court five eye" theory. Proportion of length and width of person's face is specific as follows: "three courts" represents the distance between hairline to the lower jaw. It is divided into three divisions, the first part is from the hairline to the eyebrows, the second part is from brow to the nasal floor, the third equal from the nasal floor to his chin. "Five eyes" is the distance from ear to ear to be equal to five times of the length of eyes. First of all, we draw a line in the center of the face in vertical through the frontal tip - of - the axis of the jaw, draw a horizontal line through eyebrow, a line a parallel edge of through nose. In this way, two parallel lines divide face into three parts, from the hairline to the brow wired,, from edge of the brow to the nose, from nasal alar edge to the pointed chin, each occupies one-third, which is the "three courts'. And "five eyes" refers that from the lateral canthus to the same side on the edge of the hair, the length is just one eye, length between the two eyes is also the length of the eye, the other side to the side of the hairline is an eye length. This is the "five eyes". This is the most basic standards. Two eye itself for two units, between two eye for an eye, two eyes outside the long, is composed of five eyes' length. Therefore, we can use mathematical statistical method to analyze face, namely analyze human facial data parsing from the physiology of the angle of to set up constraints formula to obtain feature points[3].

\section{Snakes algorithm}

Active contour model algorithm Snakes algorithm was proposed by Kass in 1987, can be used in boundary detection and image, segmentation, etc. The algorithm reach the purpose of detecting contour through deformation of the initial contour line of the actual contour of the target object convergence. Since move like Snakes when contour deformation, active contour model algorithm is called for Snakes. Use ordered points of images on set to define a contour model:

$$
\begin{aligned}
& v=\left\{v_{1}, \Lambda, v_{n}\right\} \\
& v_{i 1}=\left\{x_{1}, y_{1}\right\}, i=\{1, \Lambda, n\}
\end{aligned}
$$

Through an energy minimization problem, Snakes algorithm makes the iterative approximation and eventually converges at a point on the contour on the boundary of the target object. This paper studies on the contour of the $r \times r$ neighborhood $v_{i}$. For each point $p$, calculating the energy as follows:

$$
E(p)=\alpha E_{I E}(p)+\beta_{\text {ext }}(p)
$$

Improved Snakes algorithm can determine the scope of various characteristics of the part to be detected in advance, this can greatly reduce the search scope. Only in a small rectangular area to search greatly reduce the computational complexity, and the initial position is more close to the boundary of the target object. :

First of all, defines the internal energy function respectively. Active contour model is:

$$
\begin{aligned}
& v=\left\{v_{1}, \Lambda, v_{n}\right\} \\
& v_{i 1}=\left\{x_{1}, y_{1}\right\}, i=\{1, \Lambda, n\}
\end{aligned}
$$

Modify point the neighborhood of $V_{i}$ to make the contour model move in one direction. First of all, makes the contour model previously placed above the target object. Then, various points on the contour vertical move down. Neighborhood of the point is $\mathrm{rx} 1$ matrix $S_{i}$, for each point $p$ in $S_{i}$, the internal energy function is:

$$
E_{\text {int }}(p)=a E_{I E}(p)+b E_{\text {ext }}(p)
$$

Among them, the $\mathrm{a}$ and $\mathrm{b}$ are for parameters,

$$
\begin{aligned}
& E_{\text {cow }}(p)=\left\|p-\frac{v_{i-1}+v_{i+1}}{2}\right\|^{2} \\
& E_{d r}(p)=-y_{r}
\end{aligned}
$$

Calculate the neighborhood of each point of energy, adjust the minimum energy to V. This paper uses the statistics of energy maximum, minimum in the process for each energy function to 
standardize processing in order to solve the image size different influence in the field of energy. The normalized processing function is as follows:

$$
\begin{aligned}
& E(p)=a E_{\text {cow }}(p)+b E_{\text {dir }}(p) c a E_{\text {cow }}(p)+d E_{\text {cow }}(p) \\
& E_{\text {cow }}(p)=\frac{E_{\text {cow }}(p)-E_{\text {cow }}(p)_{\min }}{E_{\text {cow }}(p)_{\max }-E_{\text {cow }}(p)_{\min }} \\
& E_{\text {dir }}(p)=\frac{-y_{r}}{\text { height }} \\
& E_{\text {inten }}(p)=\frac{E_{\text {inten }}(p)-E_{\text {inten }}(p)_{\min }}{E_{\text {inten }}(p)_{\text {max }}-E_{\text {inten }}(p)_{\min }} \\
& E_{\text {grand }}(p)=\frac{E_{\text {grand }}(p)-E_{\text {grand }}(p)_{\min }}{E_{\text {grand }}(p)_{\text {max }}-E_{\text {grand }}(p)_{\text {min }}}
\end{aligned}
$$

\section{Active appearance model}

Active appearance model (AAM) is also called dynamic apparent model. Apparent here refers to two aspects, the shape and texture that is to say apparent model is built by the combination of shape model and the object of texture model. [4]AAM model of dynamic constantly adjust fitting model matching to generate accurate simulation of the model and the object. This section mainly focus on shape modeling, apparent modeling, AAM model instance to introduce positioning process of AAM model.

\section{1) Shape modeling}

The establishment of AAM shape model need first select a set of training set. Quality of the training set determines the object' basic attributes of feature point distribution of modeling. Feature points in training image tag generally adopt the method of manual marking, or semi-automatic labeling method, namely computer automatic tagging part of feature points, other anchor point is artificial, the advantages of which method is that it is efficient to figure out the initial model.

2) Apparent modeling

Active appearance model of apparent object texture information refers to the combination of shape and texture of the object. In order to obtain the normalized texture sample, texture of normalized after the CPA is conducted in the first place. Build a reversible mapping equation for each of the apparent sample, use the texture mapping method in graphics to get effective area of grain; Then, do consistent parameter sampling to map the effective to a benchmark shape texture of grid for each area. After normalization, the apparent parameters of the model is realized.

3) AAM instance generation

AAM model instance generation is also an important part of AAM. To get a description of the current object AAM model, the apparent of AAM model need to be mapped in the form of affine transformation to the shape of the corresponding instance. In the AAM fitting process, the purpose to achieve the AAM model instance with undetermined object matching is to to generate a new model through the adjustment of the corresponding fitting method, until the model instance can match the object generated.

\section{Facial cartoon animation system design}

\section{Facial cartoon animation system principle}

Facial expression animation and face cartoon image processing research method are various, but not a simple and effective implementation of facial cartoon animation function of the system. This topic is studied to solve this problem, a facial animation system is designed[5].

Facial animation system design includes two contents, facial animation and cartoon image. In facial animation design, considering the facial expression changes mainly through different states of the place such as eyebrows, eyes and mouth to express the character's emotional characteristics, so drive these parts of the drive unit by an expression data can realize the combination of different expression. In addition, in the design of face deformation, considering express man characteristics 
with humor, the facial expression exaggeration description method is adopted in the cartoon to implement cartoon of human face image.

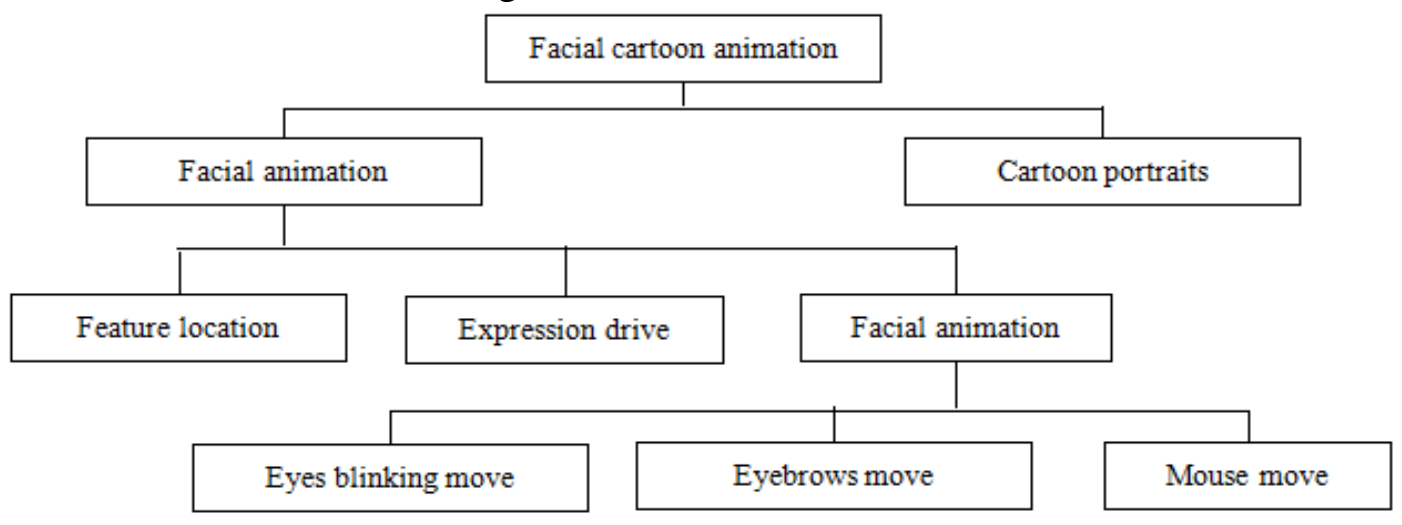

Figure 1. Facial animation system

\section{Modules of facial cartoon animation system}

Face images input are processed mainly through three modules. Firstly, enter the facial feature location module and process image through common methods of image to remove the complex background information, and then use active shape model to locate the facial feature points. The main function of this module is to find the key part of the face, including people's eyes, mouth, and eyebrows so as to provide prior conditions for implementation under the facial animation. Whether the module can accurate positioning of the key parts of the face or not directly affect the treatment effect. on the basis of the original method this article locate a key part of the face, eyes, mouth, nose, and the contours of the eyebrows with a weight limit to assist in the channeling of the facial feature location, which basically can achieve the ideal effect of positioning and meet the requirements of system design.

\section{Facial expression drive unit}

Besides the three main modules of the system, there is still a key design, the facial expression animation data drive unit. The unit's main function is to realize the facial expression of different expressions.

Face facial expression, we can have thousands of expressions, which can be summarized as six basic expressions and all other expressions are made by combing the six kinds of expression together according to certain standards. The six expressions are happy, sad, fear, anger, disgust, and surprise. From the observation it can be found that these expressions are mainly showed by forehead, brow, eyes, nose, mouth, cheeks and so on.

\section{Summary}

This article mainly focused on 2D face images modeling research and discussion. The content of the research include facial feature points positioning, facial expression animation, and deformation of the face. Some specific implementation algorithm and solution is put forward. This article discussed the face deformation algorithm and the implementation algorithm of facial expression animation existing, discussed the advantages and disadvantages of various methods, and adopted based on the physical model of face modeling under comprehensive comparative analysis, which method can match the requirement of system design in simple and effective way. In addition, modeling of the movement of the three most critical parts of the facial expression that eyes, mouth, eyebrows, especially the eyebrows, the new algorithm is proposed. The aim of this article is to develop a system of facial cartoon animation, therefore, the facial feature points positioning is studied.

\section{References}

[1] Jue Wang, Yingqing Xu, Heung-Yong Shum, Michael F. Cohen. Video Tooning. Proceedings of 
ACM SIGGRAPH 2004, 2004: 574-580

[2] Blanz, V., Basso, and Poggio C. T. Reanimating faces in images and video. Proceedings of EUROGRAPH, Annecy, 2000: 101-108.

[3] Guan Y, Chan W, Liang X, et al. Essay matting: a stroke based approach for continuous image matting [C]. Proc of Euro graphics 2006:566-570

[4] Yang M H, Knegman D and A huja N. Detecting faces in images A survey [J]. IEEE Translation on Pattern Analysis and Machine Intelligence. 2002, 24(1):35-55.

[5] Vo1ker Blanz, Thomas Veter. A Morphable Model For The Synthesis of 3D Faces. Computer Graphics, 1999, 21(15):187-194. 